\title{
CALENDAR OF EVENTS
}

This selected listing of upcoming events is offered as a service to our readers. It provides brief information on various conferences and meetings which are of more than local significance, and covers the period of approximately one year beyond the publication date of each issue. We can of course assume no responsibility for the complete accuracy of the information on which individual entries are based. Supplementary entries and updated details are incorporated with each issue, insofar as these come in the meantime to our attention.

April 10-13, 1987

Library Association (UK) University, College and Research Section Annual Study Conference: "The Shape of Things to Come: Informaton Management in the Academic Community". Location: Glasgow, Scotland, UK. Contact: Delia Taylor, Queen Mary College Library, Mile End Road, London E1 4NS, UK. Tel.: (01) 980-4811, ext. 3303 .

May $5-7,1987$

National Online Meeting. Location: New York, NY (USA). Contact: Learned Information inc., 143 old Marlton Pike, Medford, NJ 08055, USA. Tel.: (609) 654-6266.

May 7-8, 1987

Association of Research Libraries (ARL), USA, Membership Heeting. Location: Pittsburgh, PA (USA). Contact: ARL, 1527 New Hampshire Avenue NW, Washington, DC 20036, USA. Tel.: (202) 232-2466.

May $12-14,1987$

Infobase ' 87 - International Database Exhibition and Convention. Location: Frankfurt a.M., FRG. Contact: Messe Frankfurt GmbH, Bereich 12, Postfach 970126, 6000 Frankfurt a.M. 97, FRG. Tel.: (069) 7575-824. Telex: 411558 messe d. Telefax: (069) 7575-433.

May 12-14, 1987

7e Congrès sur l'information et la documentation - IDT

187: "L'espace européen de l'information". Location:

Strasbourg, France. Contact: Comité IDT 187, Association

française des documentalistes et des bibliothécaires

spécialisés, 5 avenue Franco-russe, 75007 Paris, France.

Tel.: (01) 4705-7730.

0167-8329/87/\$3.50 @ 1987, Elsevier Science Publishers B.V. (North-Holland) 
May 14-15, 1987

Symposium on Problem-Solving in Research Libraries Contributions from Library Research. Location: Madison, WI (USA). Contact: School of Libriry and Information Studies, University of Wisconsin, Helen White Hall, 600 North Park Street, Madison, WI 53706, USA. Tel.: (608) 263-2900.

May 15-21, 1987

Medical Library Association (MHA) Annual Conference. Location: Portland, OR (USA). Contact: MLA, 919 North Michigan Avenue, Suite 3208, Chicago, IL 60611, USA. Tel.: (312) 266-2456.

June 1-5, 1987

Information Resources Management - Concepts, Strategies and Applications. Location: Dubrovnik, Yu(y)slavia. Contact: Prof. Blaise Cronin, Department of Information Science, Strathclyde Business School, University of Strathclyde, Livingstone Tower, 26 Richmond Street, Glasgow G1 1XH, Scotland, UK.

June 3-5, 1987

ACM/SIGIR Conference on Research and Development in Information Retrieval. Location: New Orleans, LA (USA). Contact: Donald H. Kraft, Department of Computer Science, Louisiana State University, Baton Rouge, LA 70803, USA. Tel.: (504) 388-1495.

June 6-11, 1987

Special Libraries Association (SLA) 78th Annual Conference: "Global Information Access - Expanding Our World". Location: Anaheim, CA (USA). Contact: Conference and Exhibits Coordinator, SLA, 1700 Eighteenth Street NW, washington, DC 20009, USA. Tel.: (202) 234-4700.

June 8-12, 1987

International Association of Technological University Libraries (IATUL) 12th Biennial Conference: "Interaction Between Technological University Libraries and Industry". Location: Espoo, Finland. Contact: Prof. Elin Törnudd, Helsingin Tekniliinen Korkeakoulun, Kirjasto, Otaniteinti: i.? 9, 02150 Espoo 15, Finland. Tel.: (00) 451-2812. Telex: 121 591 tkk sf. Telefax: (00) 451-2832.

June 9-13, 1987

Jahrestagung des Vereins deutscher Bibliothekare (VDB). Location: Augsburg, FRG. Contact: VDB, Alter Postweg 120, 8900 Augsburg, FRG. Tel.: (0821) 598802 . 
June 10-16, 1987

Canadian Library Association (CLA) 42nd Annual Conference. Location: Vancouver, British Columbia, Canada. Contact: CLA, 151 Sparks Street, Ottawa, Ontario K1P 5E3, Canada. Tel.: (613) 232-9625.

June 12-14, 1987

Library Association (UK) Information Technology Group Weekend Conference: "Memories of the Future: New Library Technologies in Information Transfer, Processing and Storage". Location: Blackpool, UK. Contact: Julia Dickmann, Library Technology Centre, Polytechnic of Central London, 309 Regent Street, London W1R 8AL, UK. Tel.: (01) 580-4562.

June 14-19, 1987

5th IFSEA International Conference of Scientific Editors: "Publications as an Integral Part of Scientific Research". Location: Hamburg, FRG. Contact: Dr. W.P. Kirchner, Bundesforschungsanstalt für $\mathrm{Fischerei,} \mathrm{Information/Dokumen-}$ tation, Palmaille 9, 2000 Hamburg 50, FRG. Tel.: (040) 38905113. Telex: 0215716 bfafi d.

June 16-18, 1987

First Pacific Conference on New Information Technology for Library and Information Professionals. Location: Bangkok, Thailand. Contact: Dr. Ching-chih Chen, 1400 Commonwealth Avenue, West Newton, MA 02165, USA. Tel.: (617) 527-3431.

June 27 - July 2, 1987

American Library Association (ALA) Annual Conference. Location: San Francisco, CA (USA). Contact: ALA, 50 East Huron Street, Chicago, IL 60611, USA. Tel.: (312) 944-6780.

June 28 - July 1, 1987

Rutgers University First Annual Summer Institute for Information Professionals: "Upward Mobility - Enhancing Skills for Information Specialists". (Limited enrollment.) Location: New Brunswick, NJ (USA). Contact: Dr. Linda C. Lederman, FIPSE, Rutgers University, 4 Huntington Street, New Brunswick, NJ 08903, USA. Tël.: (201) 932-8295.

June 29 - July 1, 1987

First Conference of the International Federation of Classification Societies. Location: Aachen, FRG. Contact: IFCS 187, Prof. Dr. H. H. Bock, Institut für Statistik und Wirtschaftsmathematik, Technische Hochschule Aachen, Wüllnerstrasse 3, 5100 Aachen, FRG. 
July 11-13, 1987

International Conference on Data Bases in the Humanities and Social Sciences. Location: Montgomery, AL (USA). Contact: Dr. Lawrence J. McCrank; Dean, AUM Library and Resources Center, Auburn University at Montgomery, Montgomery, AL 36193, USA.

$$
\text { July 13-16, } 1987
$$

Institute of Information Scientists (IIS) Annual Conference: "Meeting the Challenge of Change: New Horizons for the Information Profession". Location: University of Warwick (near Coventry), UK. Contact: Jennifer Bearwood, William Kenrick Library, City of Birmingham Polytechnic, Perry Barr, Birmingham B42 2SU, UK. Tel.: (021) 356-9193, ext. 262.

July 26-31, 1987

International Association of School Librarianship Annual Conference: "The School Library - Gateway to Knowledge". Location: Iceland. Contact: Sigrun K. Hannesdottir, University of Iceland, Reykjavik, Iceland.

August 8-15, 1987

Library Automation Update. Location: London, UK. Contact: Parker Moore ltd., 10 Circus Mews, Bath BA1 2PW, UK. Tel.: (0225) 319586. Telex: 449 212. lantel g.

August 11-17, 1987

IFLA Pre-session Seminar: "Education and Training of Library, Information and Archival Personnel - Moves Towards Harmonization". Location: London, UK. Contact: IFLA, P.O.Box 95312, $2509 \mathrm{CH}$ The Hague, The Netherlands. Tel.: (070) 140884 .

August 15, 1987

IFIA Pre-session Seminar: "The Challenge of Education and Training for Information Technology". Location: Brighton, UK. Contact: Wilhelm K. Bartenbach, The H.W. Wilson Company, 950 University Avenue, The Bronx, NY 10452, USA. Tel.: (212) 588-8400.

August 17-21, 1987

53rd International Federation of Library Associations and Institutions (IFLA) General Conference: "Library and Information Services in an Evolving World". Location: Brighton, UK. Contact: Rob Palmer, Library Association, 7 Ridgmount Street, London WC1E 7AE, UK. Tel.: (01) 636-7543. Telex: 21897 laldn $g$. 
August $21-23,1987$

Library Association (UK) International and Comparative Librarianship Group Weekend Conference: "Technology for Information in Development". Location: Falmer (Brighton), UK. Contact: Diana Guthrie, Institute of Education Library, 11-13 Ridgmount Street, London WC1E 7AH, UK. Tel.: (01) 937-0846.

August 21-24, 1987

Development of Information Services in the Third Horld. Location: Brighton, UK. Contact: John Roe, College of Librarianship Wales, Llanbadarn Fawr, Aberystwyth, Dyfed, Wales SY23 3AS, UK. Tel.: (0970) 3181. Telex: 35391 clw g.

August 24-28, 1987

First International Conference on Bibliometrics and Theoretical Aspects of Information Retrieval. Location: Diepenbeek, Belgium. Contact: Prof. Dr. Leo Egghe, Bibliotheek, Limburgs Universitair Centrum, Universitaire Campus, 3610 Diepenbeek, Belgium. Tel.: (011) 229961.

August 24-28, 1987

6th National Conference on Artificial Intelligence. Location: Seattle, WA (USA). Contact: Lorraine Cooper, American Association for Artificial Intelligence, 445 Burgess Drive, Menlo Park, CA 94025, USA. Tel.: (415) $328-3123$.

September 15-18, 1987

Infodial-Videotex - Congrès-exposition international sur les banques de données et le videotex. Location: Paris (Porte Maillot), France. Contact: Infodial-Videotex '87, 4 place de Valois, 75001 Paris, France. Tel.: (01) 4261-5242. Telex: 212597 f.

September 19-27, 1987 15 th Congress of the International Association of Bibliophiles. Location: Copenhagen, Denmark. Contact: Congress AIB '87, Mr. Poul A. Christiansen, Universitetsbiblioteket, Norre Alle 49, 2200 K $\phi$ benhavn, Denmark.

September 21-24, 1987

25th International Archival Round Table Conference: "Preservation of the Archival Heritage". Location: Gardone Riviera, Italy. Contact: Mr. F.C.J. Ketelaar, Secretary, CITRA/ICA Conference, Rijksarchief, Sint Jansstraat 2, 9712 JN Groningen, The Netherlands. Tel.: (050) 127347. 
September 23-25, 1987 Deutscher Dokunentartag. Location: Bad Dürkheim, FRG. Contact: Deutsche Gesellschaft für Dokumentation, Westendstrasse 19, 6000 Frankfurt a.M. 1, FRG. Tel.: (0611) 747761 .

October 4-8, 1987

50 th American Society for Information Science (ASIS) Annual Meeting. Location: Boston, MA (USA). Contact: ASIS, 1424 Sixteenth Street NW, Suite 404, Washington, DC 20036, USA. Tel.: (202) 462-1000.

October 6-9, 1987

Public Library Association (UR) Conference. Location: Torquay, UK. Contact: Harry Galloway, Avon County Library Headquarters, College Green, Bristol BS1 5TL, UK. Tel.: (0272) 276121, ext. 248. Telex: 44200.

October 13-15, 1987

European Association of Information Services (EUSIDIC)

Annual Conference. Location: Lausanne, Switzerland. Contact: EUSIDIC Administrative Secretariat, P.0.Box 429, London W4 1UJ, UK. Tel.: (01) 546-7968. Telex: 32.5962.

October 20-22, 1987

Online '87. Location: Anaheim (Disneyland), CA (USA). Contact: Online inc., 11 Tannery Lane, Weston, CT 06883, USA. Tel.: (203) 227-8466.

October 22-23, 1987

Association of Research Libraries (ARL), USA, Membership Meeting. Location: Washington, DC (USA). Contact: ARL, 1527 New Hampshire Avenue NW, Washington, DC 20036, USA. Tel.: (202) 232-2466.

October 27-30, 1987

Educom '87. Location: Los Angeles, CA (USA). Contact: Carol Parysz, Educom, P.O.Box 364, Princeton, NJ 08540, USA. Tel.: (609) $734-1888$.

December 8-10, 1987

11th International Online Information Meeting. Location: London, UK. Contact: Learned Information ltd., Woodside, Hinksey Hill Oxon. 0X1 5AU, UK. Tel.: (0865) 730275. Telex: 837704 inform g. Telefax: (0865) 736354 . 


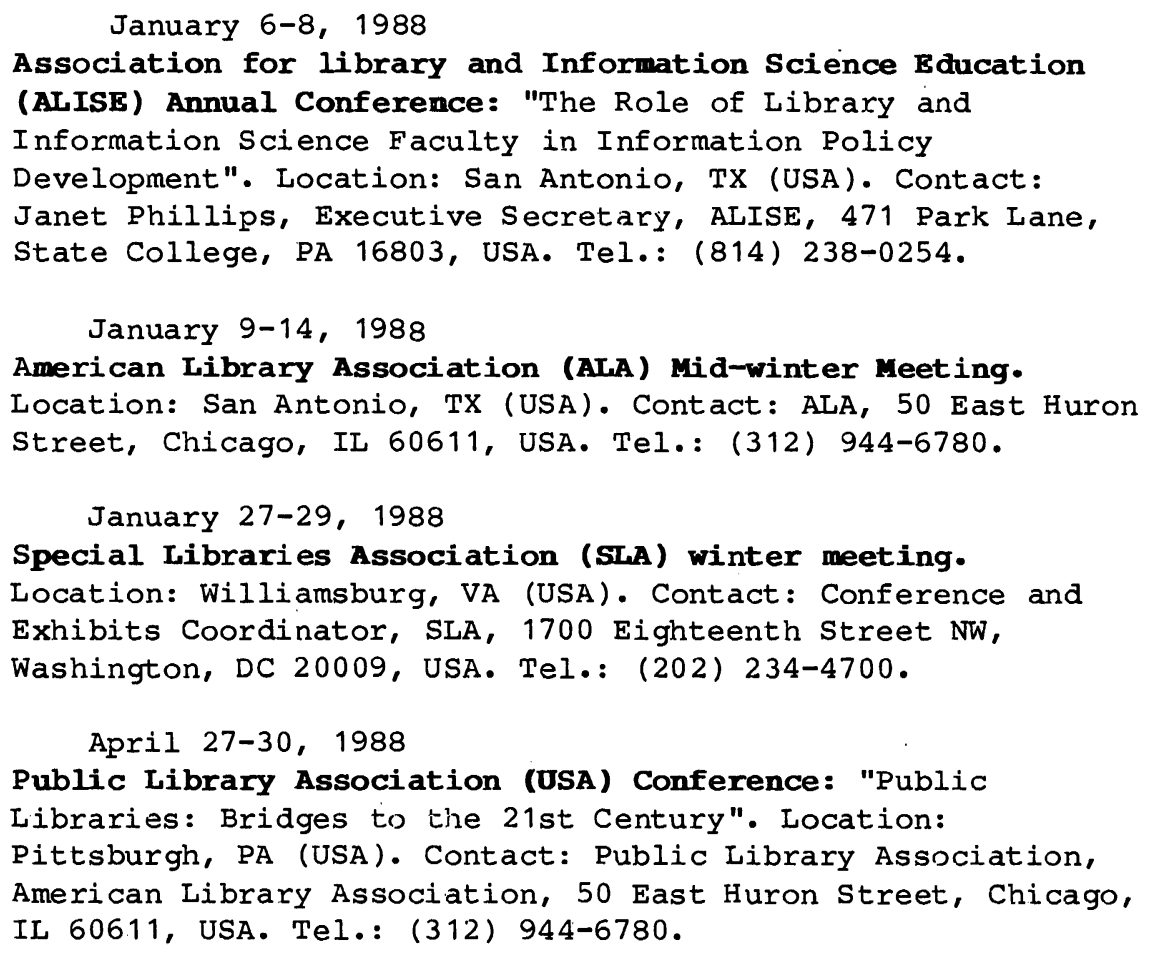

